\title{
Intraocular pressure and pulsatile ocular blood flow after retrobulbar and peribulbar anaesthesia
}

\author{
Russell Watkins, Bijan Beigi, Mark Yates, Bernard Chang, Evangelos Linardos
}

\begin{abstract}
Aims-This study investigated the effect of peribulbar and retrobulbar local anaesthesia on intraocular pressure (IOP) and pulsatile ocular blood flow (POBF), as such anaesthetic techniques may adversely affect these parameters.

Methods-20 eyes of 20 patients who were to undergo phacoemulsification cataract surgery were prospectively randomised to receive peribulbar or retrobulbar anaesthesia. The OBF tonometer (OBF Labs, Wiltshire, UK) was used to simultaneously measure IOP and POBF before anaesthesia and 1 minute and 10 minutes after anaesthesia. Between group comparisons of age, baseline IOP, and baseline POBF were performed using the non-parametric Mann-Whitney test. Within group comparisons of IOP and POBF measured preanaesthesia and post-anaesthesia were performed using the non-parametric Wilcoxon signed ranks test for both groups. Results-There was no statistically significant IOP increase post-anaesthesia in either group. In the group receiving peribulbar anaesthesia, there was a significant reduction in POBF initially postanaesthesia which recovered after 10 minutes. In the group receiving retrobulbar anaesthesia, there was a persistent statistically significant reduction in POBF.
\end{abstract}

Conclusions-Retrobulbar and peribulbar injections have little effect on IOP. Ocular compression is not needed for IOP reduction when using local anaesthesia for cataract surgery. Conversely, POBF falls, at least for a short time, when anaesthesia for ophthalmic surgery is administered via a retrobulbar route or a peribulbar route. This reduction may be mediated by pharmacologically altered orbital vascular tone. It may be safer to use other anaesthetic techniques in patients with ocular vascular compromise.

(Br F Ophthalmol 2001;85:796-798)

Ophthalmology, Leeds

General Infirmary,

Clarendon Wing,

Belmont Grove, Leeds

LS2 9NS, UK

B Chang

Correspondence to: Dr Russell Watkins, Department of Optometry, Anglia Polytechnic

University, East Road,

Cambridge CB1 1PT, UK

r.j.watkins@apu.ac.uk

Accepted for publication

14 February 2001 anaesthesia techniques can lead to an increase in intraocular pressure (IOP), which can be countered by using ocular compression such as that which can be obtained by the use of such devices as Honan's balloon. ${ }^{1}$

The situation is not as clear regarding changes in ocular blood flow with different methods of local anaesthesia. A reduced ocular blood flow, with or without a raised IOP, may be hazardous to patients. This is especially so if the ocular circulation is already compromised such as in cases of glaucoma or ischaemic optic neuropathy.

It is possible to measure pulsatile ocular blood flow (POBF) using pneumotonographic techniques. The pulsatile component of ocular blood flow is primarily choroidal. ${ }^{2}$ Since choroidal blood flow is ultimately derived from the same source as the optic nerve head (that is, the short posterior ciliary arteries) it may be that changes in choroidal blood flow reflect changes in optic nerve head blood flow.

The aim of this study was to investigate the effect of peribulbar and retrobulbar local anaesthesia on IOP and POBF.

\section{Patients and methods}

PATIENT CHARACTERISTICS

Twenty eyes of 20 patients who were to undergo planned phacoemulsification cataract surgery were prospectively randomised to have their surgery carried out under peribulbar $(\mathrm{n}=$ 10; four males and six females) or retrobulbar anaesthesia ( $\mathrm{n}=10$; four males and six females). Patients with a history of vascular hypertension diabetes mellitus, any form of glaucoma, or ocular hypertension (Goldmann applanation IOP $>24 \mathrm{~mm} \mathrm{Hg}$ when sitting) and previous intraocular surgery were excluded. Furthermore, none of the patients enrolled in this study had myopia $>4$ dioptres equivalent sphere. Informed consent was obtained and this research was performed with respect for the tenets of the Declaration of Helsinki.

LOCAL ANAESTHETIC AND MEASUREMENT TECHNIQUES

The OBF tonometer (OBF Laboratories, Wilts, UK) measures IOP and POBF simultaneously. A disposable probe is used to applanate the cornea for a maximum of 10 seconds and the IOP and POBF measurements are taken during this time. The probe is connected to a central processing unit. The OBF tonometer uses the principle of pneumotonography to measure IOP and the magnitude of the ocular pulse and, with certain valid assumptions, simultaneously calculates the POBF. $^{3}$ POBF primarily reflects pulsatile choroidal arterial inflow. ${ }^{2}$ The technique is easily applied, objective, repeatable, and reliable with a single measurement being representative of $\mathrm{POBF}^{4}$ 


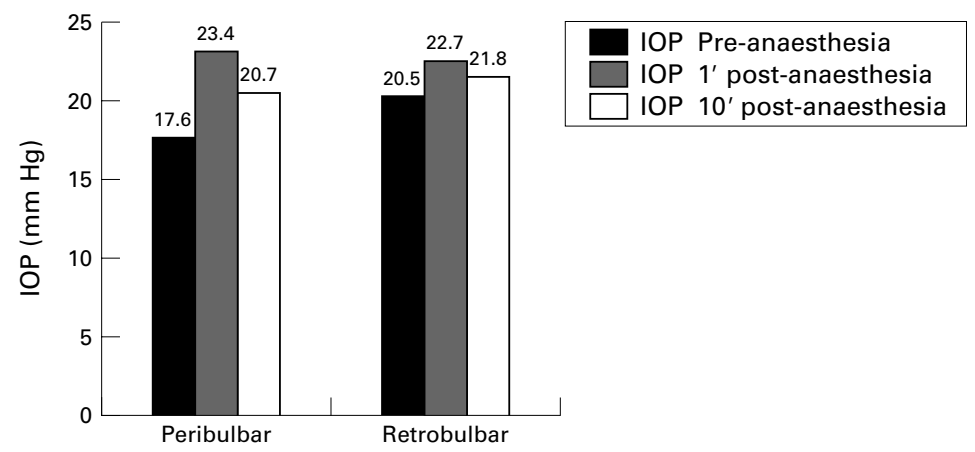

Figure 1 Median intraocular pressures (IOP) pre-anaesthesia and post-anaesthesia for both groups. See text for details.

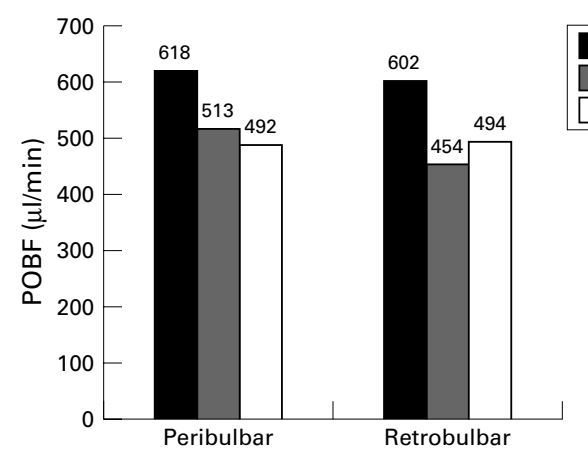

Figure 2 Median pulsatile ocular blood flow (POBF) values pre-anaesthesia and post-anaesthesia for both groups. See text for details.

All eyes received a preoperative dilating eye drop regimen of either cyclopentolate $1 \%$, phenylephrine $10 \%$, diclofenac $0.1 \%$, and amethocaine $1 \%$; or cyclopentolate $1 \%$, phenylephrine $2.5 \%$, flurbiprofen $0.03 \%$, and amethocaine $1 \%$ over $2-3$ hours. All measurements were taken after instillation of amethocaine $1 \%$ eye drops with the patient lying flat. (Note that some patients seemingly had IOPs outside our criteria-this may be because measurements were taken supine, with the pupil dilated.) IOP and POBF were measured immediately before the peribulbar or retrobulbar injection and then at 1 minute and again at 10 minutes following the anaesthetic injection.

For peribulbar anaesthesia, a mixture of equal amounts of lignocaine $2 \%$, bupivacaine $0.75 \%$, with 150 units of hyaluronidase was prepared and $10 \mathrm{ml}$ injected into the peribulbar space transconjunctivally, inferotemporally, and medially. For retrobulbar anaesthesia, a mixture of equal amounts of lignocaine $2 \%$, bupivacaine $0.75 \%$, with 150 units of hyaluronidase was prepared and $3 \mathrm{ml}$ injected into the intraconal space. No oculocompressive devices were used in either group.

\section{STATISTICAL ANALYSIS}

All statistical comparisons were performed using sPSs 9.0 for Windows (SPSS Inc). Between group comparisons of age, baseline IOP, and baseline POBF were performed using the non-parametric Mann-Whitney test. Within group comparisons of IOP and POBF measured at the three different times were performed using the non-parametric Wilcoxon signed ranks test both for the group receiving peribulbar anaesthesia and the group receiving retrobulbar anaesthesia. While POBF values are probably normally distributed within the population, ${ }^{4}$ non-parametric statistics were used because of the relatively small sample size.

\section{Results}

The median measurements of IOP and POBF are shown graphically in Figures 1 and 2 . Those patients who received peribulbar anaesthesia had a median age of 79 years (range 58-91 years) while those who received retrobulbar anaesthesia had a median age of 80 years (range 68-89). The age distributions were not significantly different between the two groups (Mann-Whitney $\mathrm{U}=47.5 ; \mathrm{p}=\mathrm{ns}$ ). Baseline (that is, pre-anaesthetic) IOPs were similar between the two groups (MannWhitney $\mathrm{U}=46.0 ; \mathrm{p}=\mathrm{ns}$ ) as were baseline POBF measurements (Mann-Whitney $\mathrm{U}=$ 49.5; $\mathrm{p}=\mathrm{ns}$ ).

Although IOP increased 1 minute after injection in both the peribulbar and retrobulbar anaesthesia groups, this increase was not statistically significant. The IOP decreased towards, but did not reach, the baseline IOP in both groups at 10 minutes. The residual increase was again not statistically significant in either group.

In the group receiving peribulbar anaesthesia, there was a significant fall in POBF 1 minute after the injection of anaesthetic (Wilcoxon $\mathrm{Z}=-2.29$; $\mathrm{p}=0.022$ ). POBF was also lower in this group 10 minutes following the injection of anaesthetic but this fall was no longer significantly different from baseline (Wilcoxon $\mathrm{Z}=-1.60 ; \mathrm{p}=\mathrm{ns}$ ).

In the group receiving retrobulbar anaesthesia, there was a statistically significant fall in POBF at 1 minute post-injection (Wilcoxon $\mathrm{Z}$ $=-2.50 ; \mathrm{p}=0.013)$ which persisted at $10 \mathrm{~min}-$ utes following anaesthetic injection (Wilcoxon $Z=-2.80 ; p=0.005)$.

\section{Discussion}

In this study, we have demonstrated that POBF falls, at least for a short time, when anaesthesia for ophthalmic surgery is administered via a retrobulbar route or a peribulbar route. We observed a statistically significant fall in POBF 1 minute after peribulbar and retrobulbar anaesthetic injections. This reduction in POBF persisted (at 10 minutes) in the group receiving retrobulbar injections.

In the context of this study, ocular blood flow can be perturbed by three mechanisms: (1) pharmacologically mediated changes in vascular calibre, (2) compression, by the anaesthetic bolus, of the retrobulbar and peribulbar vasculature, and (3) an anaesthetic bolus induced increase in intraocular pressure. Pharmacological effects can arise from the preoperative dilatation regimen (for instance, cyclopentolate and phenylephrine have vasoconstrictive effects), from the instillation of amethocaine eye drops (amethocaine is a mild vasodilator) and from the peribulbar or retrobulbar anaesthetic mixtures. For instance, Meyer et $a \bar{l}$ showed that bupivacaine can impair nitric oxide mediated vasodilatation in 
porcine ciliary arteries. Within a few minutes of injection, peribulbar anaesthesia produces a similar distribution of local anaesthetic mixture to retrobulbar anaesthesia within the confined tissue space of the orbit. ${ }^{6}$ If the vasomotor effects of the anaesthetic mixtures were significant, one would expect similar effects on POBF in the group receiving peribulbar anaesthesia and the group receiving retrobulbar anaesthesia. In our sample, this feature was observed. Anaesthetic induced change of vascular tone may therefore cause changes in POBF. Pharmacologically induced changes in vascular tone have been implicated in reducing ocular blood flow in other studies. ${ }^{78}$ The wide spread of normal POBF values which has been previously documented ${ }^{4}$ was confirmed in this study. This spread in a small sample may explain the apparent paradox of why there was no observed improvement in POBF values at 10 minutes in the peribulbar group, although the median POBF was not significantly different from baseline POBF.

Hulbert and colleagues ${ }^{7}$ demonstrated that hyaluronidase added to retrobulbar injections of lignocaine appears to lessen the reduction in POBF, putatively by reducing the mass effect of the anaesthetic mixture. However, the IOP may still rise owing to the increase in retrobulbar pressure associated with a bolus of anaesthetic mixture. This study did not demonstrate a statistically significant change in IOP following either retrobulbar or peribulbar anaesthesia. However, it is possible that bolus induced changes in IOP influenced POBF in this study but the sample was too small for a meaningful analysis of this effect. Other studies, however, have also not shown IOP changes. ${ }^{79}$ The mechanism for a fall in POBF associated with increasing IOP is presumably by obstruction of venous drainage and a reduction in perfusion pressure. It is notable in this respect that vascular autoregulation is poor or absent in the choroid ${ }^{10}$ and it is the choroidal blood flow that is the principal component of POBF measurement by pneumotonometry. The findings of this study may contradict those of Findl $e t a l^{8}$ who demonstrated a sustained reduction in ocular blood flow after peribulbar anaesthesia, an effect they attributed to changes in orbital vascular tone. However, this was at 5 minutes after local anaesthesia, whereas we measured POBF 10 minutes later, which may have been enough time to allow for recovery of POBF. Findl et al also used laser interferometry and Doppler ultrasound to characterise ocular blood flow.

A recent study by Chang and colleagues, of similar design to the present study, has demonstrated that subconjunctival anaesthesia produces no significant change in either IOP or POBF. $^{9}$ They also showed that peribulbar anaesthesia with or without balloon compression produced significant falls in POBF after local anaesthesia and that in the group without Honan's balloon compression, POBF remained significantly low at 10 minutes. ${ }^{9}$ The present study, where median POBF at $10 \mathrm{~min}-$ utes was not significantly different from baseline in the group receiving peribulbar anaesthesia, contradicts these findings. However, there was a trend towards a decreased POBF (see Fig 2) and the small sample size may have hidden a true effect.

The clinical relevance of these findings lie in the possible ocular damage resulting from periods of relative ocular ischaemia (and from ischaemia reperfusion injury) brought about by reductions in POBF. Patients with already compromised ocular circulation-for example, those with glaucoma, may be more at risk when ocular blood flow falls. However, vascular events following anaesthetic injection are rare, ${ }^{11}$ possibly because total ocular blood flow, across systole and diastole may be constant. ${ }^{12}$ Retrobulbar anaesthesia is becoming less popular and topical anaesthesia is gaining popularity which should negate these adverse effects.

In summary, ocular compression-for instance, using Honan's balloon, is seemingly not needed for IOP reduction when using local anaesthesia for cataract surgery. It is assumed that because there is a smaller volume of anaesthetic mixture injected with retrobulbar anaesthesia compared with peribulbar anaesthesia, there will be a smaller rise in IOP; this assumption does not seem to be valid as both retrobulbar and peribulbar injections produce similar effects on IOP. From the point of view of ocular perforation, peribulbar anaesthesia may be safer but there is still a significant fall in POBF causing potential problems for the patient with ocular vascular compromise. Other anaesthetic techniques (that is, topical or subconjunctival anaesthesia) might be safer in these patients.

We are grateful to our patients for consenting to participate in this study.

Competing interests: None.

1 O’Donoghue E, Batterbury M, Lavy T. Effect on intraocular pressure of local anaesthesia in eyes undergoing intraocular surgery. Br f Ophthalmol 1994;78:605-7.

2 Langham ME, Farrell RA, O'Brien V, et al. Non-invasive measurements of pulsatile blood flow in the human eye. In: Lambrou GN, Greve EL, eds. Ocular blood flow in glaucoma. Amsterdam: Kugler and Ghedini, 1989:93-9.

3 Silver DM, Farrell RA. Validity of pulsatile ocular blood flow measurements. Surv Ophthalmol 1994;38(Suppl): s72-80.

4 Yang YC, Hulbert MFG, Batterbury M, et al. Pulsatile ocular blood flow measurements in healthy eyes: reproduclar blood flow measurements in healthy eyes: reprod

5 Meyer P, Flammer J, Luscher TF. Local anesthetic drugs reduce endothelium-dependent relaxation of porcine ciliary arteries. Invest Ophthalmol Vis Sci 1993;34:2730-6.

6 Ropo A, Nikki P, Ruusuvaara P, Kivisaari L. Comparison of retrobulbar and periocular injections of lignocaine by computerised tomography. $\mathrm{Br} \mathcal{F}$ Ophthalmol 1991;75:417-20.

7 Hulbert MFG, Yang YC, Pennefather PM, et al. Pulsatile ocular blood flow and intraocular pressure during retrobulbar injection of lignocaine: influence of additives. $\mathcal{F}$ Glaucoma 1998;7:413-16.

8 Findl O, Dallinger S, Menapace R, et al. Effects of peribulbar anesthesia on ocular blood flow in patients undergoing cataract surgery. Am f Ophthalmol 1999;127:645-9.

9 Chang BYP, Hee WCL, Ling R, et al Local anaesthetic Chang BYP, Hee WCL, Ling R, et al Local anaesthetic
techniques and pulsatile ocular blood flow. Br f Ophthalmol 2000;84:1260-3.

10 Alm A. Ocular circulation. In: Hart WM, ed. Adler's physiology of the eye. 9th ed. St Louis: Mosby, 1992:215.

11 Ruben S. The incidence of complications associated with retrobulbar injection of anesthetic for ophthalmic surgery. Acta Ophthalmol Copenh 1992;70:836-8.

12 Jay WM, Aziz MZ, Green K. Further studies on the effect of retrobulbar epinephrine injection on ocular and optic nerve blood flow. Curr Eye Res 1985;4:55-8. 